\title{
Improved Solubility and Bioactivity of Camptothecin Family Antitumor Drugs with Supramolecular Encapsulation by Water- Soluble Pillar[6]arene
}

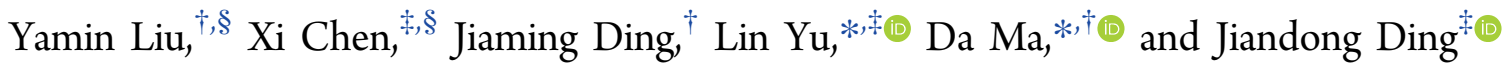 \\ ${ }^{\dagger}$ Department of Chemistry and ${ }^{\ddagger}$ State Key Laboratory of Molecular Engineering of Polymers, Department of Macromolecular \\ Science, Fudan University, 220 Handan Road, Shanghai 200433, China
}

Supporting Information

\begin{abstract}
Water-soluble pillar[6]arene (WP6) was used to solubilize camptothecin family antitumor drugs. In the presence of WP6, the solubility of camptothecin (CPT) and 10-hydroxycamptothecin (HCPT) was enhanced by 380 and 40 times, respectively. The solubility enhancement is proved to be the result of the host-guest encapsulation by WP6. WP6 has a low cytotoxicity against normal MC 3T3-E1 cells, whereas the bioactivity of CPT and HCPT is substantially improved as a result of the solubility enhancement.
\end{abstract}
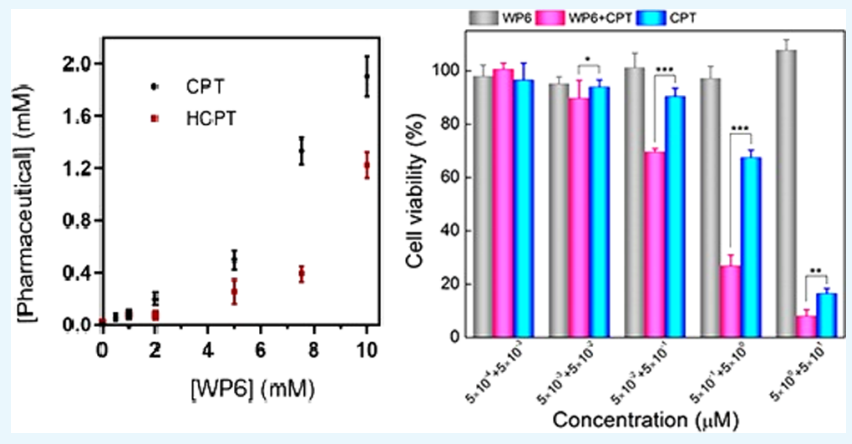

\section{INTRODUCTION}

Discovered in 1966, 20-(S)-camptothecin (CPT) and its derivatives constitute a family of important antitumor drugs. ${ }^{1}$ CPT shows powerful antitumor activity by targeting DNA topoisomerase I (TOP I). ${ }^{2}$ However, CPT suffers from two major limitations: (1) CPT is sparingly soluble in water, limiting its clinical applications via intravenous injection; (2) the biologically active form of CPT (lactone) rapidly undergoes a ring-opening reaction and converts to the biologically inactive form (carboxylate) under neutral conditions (Figure 1a). ${ }^{3} \mathrm{CPT}$ derivatives with improved solubility in water have been developed, including the clinically approved antitumor drugs topotecan and irinotecan (Figure 1b). ${ }^{4,5}$ Liposomes, ${ }^{6}$ inorganic nanoparticles, ${ }^{7}$ hydrogels, ${ }^{8}$ and prodrug conjugates ${ }^{9}$ have been developed to formulate and deliver CPT. To achieve the full potential of CPT for cancer treatment, new delivery methods are highly desirable to solubilize this hydrophobic antitumor drug.

Pillar $[n]$ arenes $(\operatorname{PA}[n] \mathrm{s}, n=5-10)$ are a type of macrocyclic hosts with a unique pillar-shaped architecture. ${ }^{10}$ Since 2008 , the excellent host-guest recognition ability of $\mathrm{PA}[n] \mathrm{s}$ has attracted great interests. ${ }^{11-18}$ Water-soluble PA $[n] \mathrm{s}$ with either anionic (carboxylate) $^{19}$ or cationic (amine) $)^{20}$ modifications have been used for drug delivery, ${ }^{21}$ material assembly, ${ }^{22}$ and antibiotic applications. ${ }^{23}$ Among these water-soluble PA $[n]$ s, carboxylated PA[6] (WP6, Figure 1c) is an important macrocyclic host with a large cavity $(7.7 \AA),{ }^{24}$ excellent host-guest recognition ability, and relatively simple synthesis. ${ }^{25}$ Recent study also demonstrated the satisfactory biocompatibility of WP6. ${ }^{26}$ There are reports of cyclodextrin and cucurbituril-based molecular containers used to formulate and solubilize hydrophobic pharmaceuticals. $^{27-31}$ The clinical success of $\beta$-cyclodextrinbased Captisol proves that solubility enhancement by hostguest encapsulation has practical use. ${ }^{32,33}$ Water-soluble PA $[n] \mathrm{s}$ have outstanding recognition property and can be easily functionalized for "smart" drug delivery. We envision that WP6 and other water-soluble PA $[n]$ s may be ideal candidates for solubility enhancement applications.

Herein, we report the use of WP6 for the solubility and bioactivity enhancement of CPT and 10-hydroxycamptothecin (HCPT). In this article, we study the WP6-assisted solubility improvement of CPT and HCPT. The recognition behavior of WP6 toward CPT family drugs is investigated. Finally, the bioactivity of WP6-solubilized CPT and HCPT, as well as the biocompatibility of WP6, are tested in vitro.

\section{RESULTS AND DISCUSSION}

2.1. Design and Synthesis. WP6 was chosen as the drug carrier for two reasons: (1) the cavity is large enough to accommodate CPT and its derivatives; (2) PA[6], the precursor to synthesize WP6, is relatively easy to prepare. WP6 was synthesized according to the literary procedures (Figure 1c). ${ }^{25}$ Starting from inexpensive materials, the condensation reaction of 1,4-diethyoxybenzene and paraformaldehyde delivered $\mathrm{PA}[6]$ with a yield of $15 \%$. After removing ethoxy groups with $\mathrm{BBr}_{3}$, phenolic hydroxyl groups were substituted with methyl esters. Finally, the hydrolysis reaction converted esters to carboxylates. The treatment with $\mathrm{NaOH}$

Received: July 20, 2017

Accepted: August 22, 2017

Published: August 31, 2017 
(a)

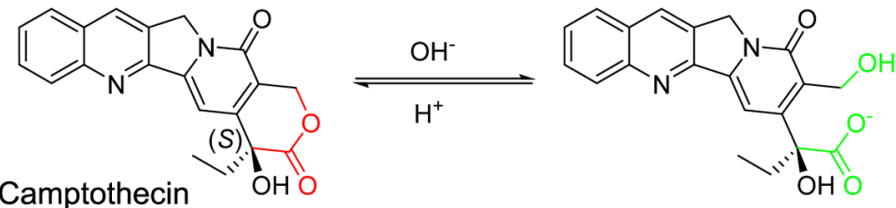

lactone

Active form

(b)<smiles>CC[C@]1(O)C(=O)CCc2c1cc1n(c2=O)Cc2cc3cc(O)ccc3nc2-1</smiles>

10-hydroxycamptothecin

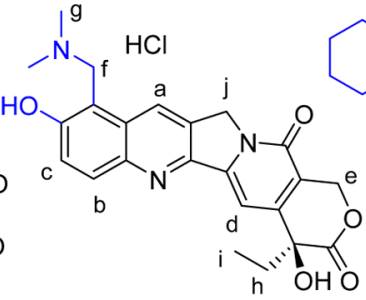

Topotecan

Inactive form

(c)

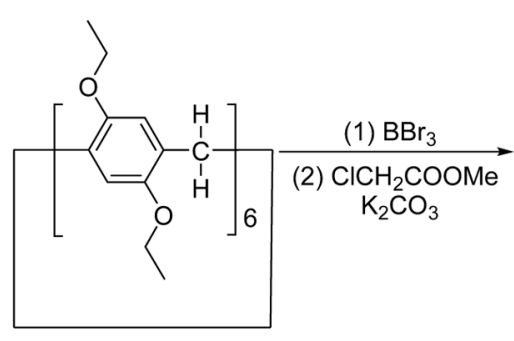

$\mathrm{PA}[6]$

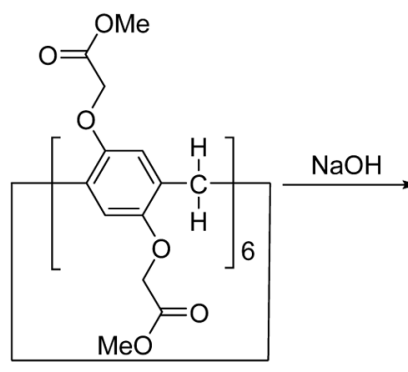

inotecan<smiles>CCc1c2c(nc3ccc(OC(=O)N4CCC(N(C)CC)CC4)cc13)-c1cc3c(c(=O)n1C2)COC(=O)[C@]3(O)[C@H](CC)OCc1ccccc1</smiles>

blue); (c) the synthesis of water-soluble pillar[6] arene (WP6).

yielded WP6 as a sodium carboxylate. The synthesis of WP6 is relatively simple, with low cost, which renders the large-scale preparation and pharmaceutical applications possible. WP6 is highly soluble in water. Its solubility was measured to be 33 $\mathrm{mM}$ in $\mathrm{NaD}_{2} \mathrm{PO}_{4}$ buffer $(10 \mathrm{mM}, \mathrm{pD}=7.4)$. Its excellent solubility in water enables WP6 to be used for solubility enhancement of hydrophobic pharmaceuticals.

2.2. Solubility Enhancement. The solubility enhancement assay was performed with poorly soluble drugs CPT and HCPT. Although CPT is barely soluble $(5 \mu \mathrm{M})$ in phosphatebuffered saline (PBS), HCPT is slightly more soluble $(29 \mu \mathrm{M})$. To determine the solubility enhancement, a known concentration of WP6 was stirred with excess solid drug for $3 \mathrm{~h}$ in $\mathrm{NaD}_{2} \mathrm{PO}_{4}$ buffer $(10 \mathrm{mM}, \mathrm{pD} 7.4)$. The excess insoluble drug was removed with centrifugation. The concentration of the drug in the supernatant was determined by ${ }^{1} \mathrm{H}$ NMR integration of drug resonances versus 1,3,5-benzenetricarboxylic acid, subsequently added as internal reference (Figures S1S12). The solubility enhancement assay was performed in triplicate. Phase solubility diagrams are shown in Figure 2. WP6 substantially improves the solubility of both drugs (380 times for $\mathrm{CPT}$ and 40 times for $\mathrm{HCPT}$ ). The solubility reaches 1.9 $\mathrm{mM}$ for CPT and $1.2 \mathrm{mM}$ for HCPT in the presence of $10 \mathrm{mM}$ WP6, which is soluble enough for dosing via intravenous injection. Both solubility phase diagrams are referred to as $A_{P}$ type plots, signifying the presence of a higher order complex (e.g. (WP6) $\left.)_{2}-\mathrm{CPT}\right)$. $^{3}$

2.3. Host-Guest Chemistry. We investigated the hostguest interaction between WP6 and CPT family pharmaceuticals. The intrinsic solubility of CPT and HCPT is too low to determine the host-guest chemistry at neutral $\mathrm{pH}$. Instead, a

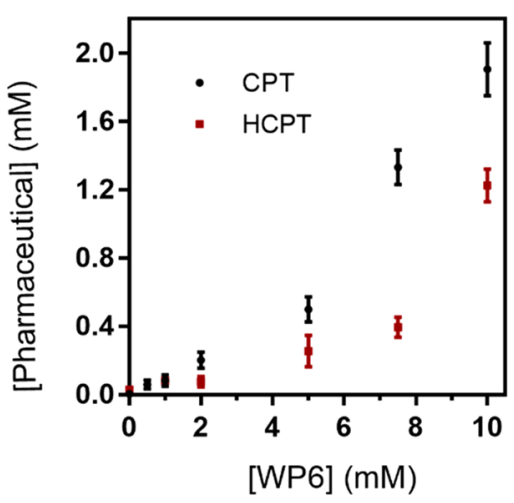

Figure 2. Phase solubility diagrams for CPT (black) and HCPT (red) with WP6. Error bars are derived from triplicates.

basic solvent of $0.1 \mathrm{M} \mathrm{NaOD}$ in $\mathrm{D}_{2} \mathrm{O}$ was used to improve the solubility of CPT (Figure S13). The ${ }^{1} \mathrm{H}$ NMR spectrum recorded for equal molarities of WP6 and CPT shows a broadening of CPT aromatic resonances, which indicates the host-guest interaction between WP6 and CPT (carboxylate form). To investigate the encapsulation under neutral condition, topotecan (TPT), a water-soluble and clinically approved CPT family drug, was used to study its binding with WP6 at $\mathrm{pH}$ 7.4. Figure 3 a shows the ${ }^{1} \mathrm{H}$ NMR spectra recorded for TPT, WP6, and mixtures of WP6 and TPT (1:2, 1:1, and 1:0.5). Aromatic proton a and methyl proton $\mathrm{g}$ undergo significant upfield shifts in the presence of WP6, which indicates the encapsulation of TPT. The resonances of upfield-shifted aromatic protons are broadened in the presence of excess TPT, without the observation of free topotecan 
(a)
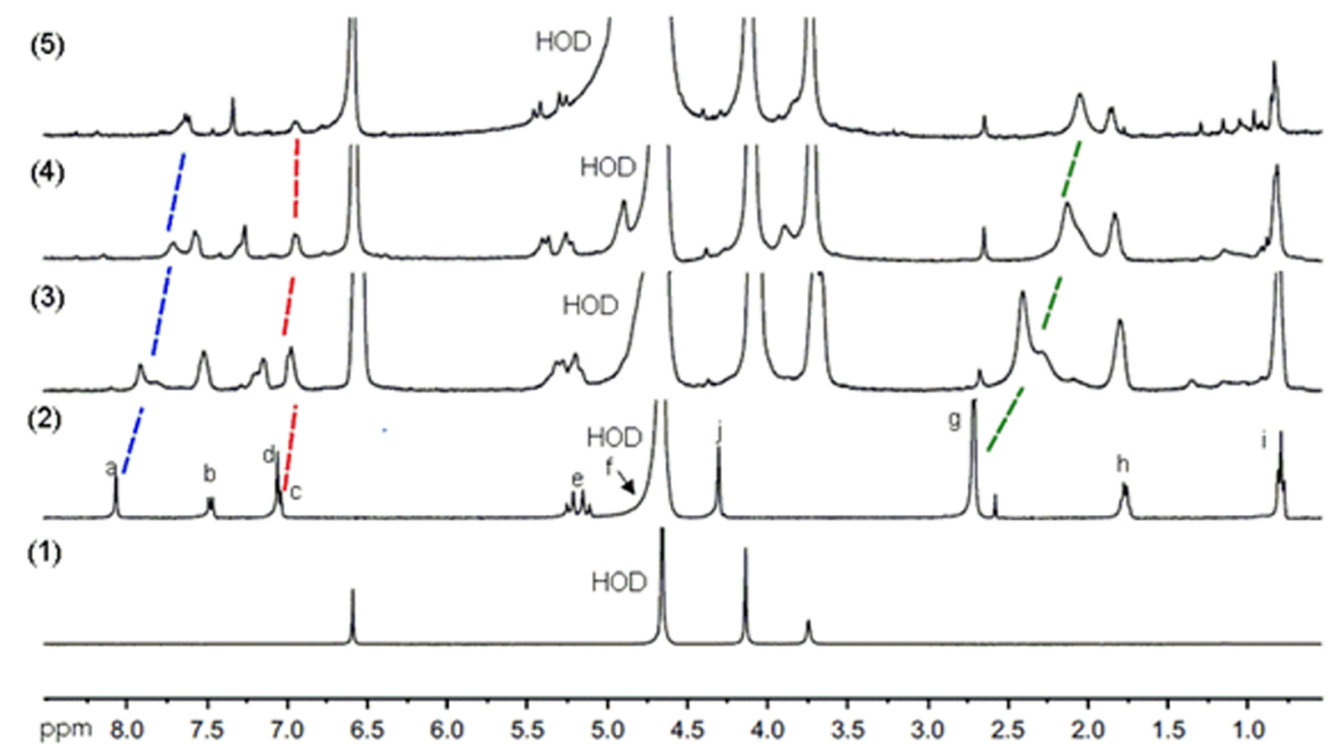

(b)<smiles>Nc1ccc2cc3ccc(N)cc3nc2c1</smiles><smiles>Cc1cc2nc3ccc(N(C)C)cc3nc2cc1N</smiles>
neutral red $\bullet \mathrm{HCl}$ (c)

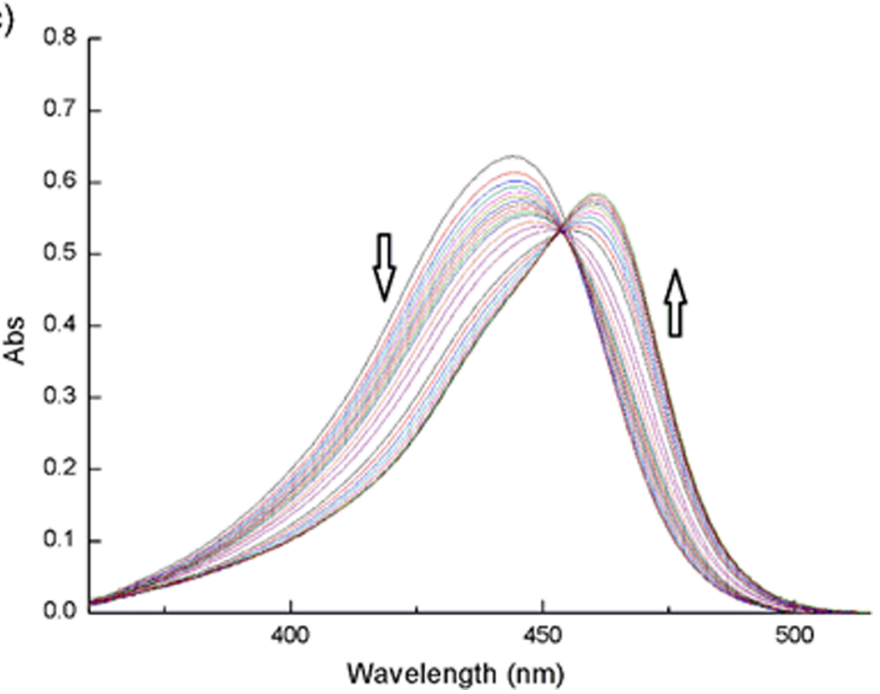

Figure 3. (a) ${ }^{1} \mathrm{H}$ NMR spectra recorded for (1) WP6, (2) topotecan, and mixtures of WP6 and topotecan, with ratios of 1:2 (3), 1:1 (4), and 1:0.5 (5) (400 MHz, room temperature, $10 \mathrm{mM} \mathrm{NaD}_{2} \mathrm{PO}_{4}, \mathrm{pD} 7.4$, [WP6] = $1 \mathrm{mM}$ ). (b) The molecular structure of dyes used in this paper, and (c) UVvis spectra from the titration of proflavine $(10 \mu \mathrm{M})$ with WP6 $(0-72 \mu \mathrm{M})$ at $\mathrm{pH} 7.4$ (PBS).

resonances, demonstrating a fast exchange on the chemical shift time scale. TPT has a $\left(\mathrm{CH}_{3}\right)_{2} \mathrm{NCH}_{2}-$ substituent, which enhances its electrostatic interaction with WP6. Parts of the TPT aromatic system are encapsulated inside the cavity, which implicates that hydrophobicity and $\pi-\pi$ stacking are also the causes of encapsulation. Unlike TPT, there is no electrostatic interaction between WP6 and CPT at basic $\mathrm{pH}$. We believe that the driving force for the host-guest interaction between WP6 and CPT (carboxylate form) under basic condition is hydrophobicity and $\pi-\pi$ stacking.

The stability of the complex formed by WP6 and the pharmaceutical is critical for the clinical use. We evaluated the complex stability by measuring the binding constant $K_{\mathrm{a}}$. Phosphate-buffered saline (PBS) was used to mimic the neutral physiological environment ( $\mathrm{pH}$ 7.4). Because CPT and HCPT are poorly soluble in water, direct ${ }^{1} \mathrm{H}$ NMR titration could not be used to determine the value of $K_{\mathrm{a}}$. Instead, we used the indicator displacement assay. ${ }^{34}$ We first measured the value of $K_{\mathrm{a}}$ for the complex of WP6 and dye. Two dyes, proflavine and neutral red, were used in the study (Figure $3 \mathrm{~b}$ ). The value of $K_{\mathrm{a}}$ for the complex of WP6 and dye was determined by direct $\mathrm{UV}-$ vis titration (Figures $3 \mathrm{c}$ and S15a). The existence of the isosbestic point shows a $1: 1$ binding stoichiometry for both dyes. We also used the Job plot to confirm that the binding stoichiometry for proflavine is 1:1 (Figure S16). The $K_{\mathrm{a}}$ value for the complex of WP6 and proflavine/neutral red was calculated to be $(1.4 \pm 0.1) \times 10^{5} \mathrm{M}^{-1}$ and $(1.3 \pm 0.1) \times 10^{5}$ $\mathrm{M}^{-1}$, respectively (Figures $\mathrm{S} 14 \mathrm{~b}$ and $\mathrm{S} 15 \mathrm{~b}$ ). We then used the indicator displacement assay by fluorescence or UV-vis spectroscopy to determine the $K_{\mathrm{a}}$ value for the complex of WP6 and CPT family antitumor drugs. The $K_{\mathrm{a}}$ value was determined to be $(8.4 \pm 1.0) \times 10^{6} \mathrm{M}^{-1}$ for CPT, $(1.2 \pm 0.2) \times$ $10^{5} \mathrm{M}^{-1}$ for HCPT, and $(1.3 \pm 0.1) \times 10^{4} \mathrm{M}^{-1}$ for TPT (Figures S17-S19). The $K_{\mathrm{a}}$ value for the complex of WP6 and 
$\mathrm{CPT} / \mathrm{HCPT}$ falls in the range of $10^{5}-10^{7} \mathrm{M}^{-1}$ in $\mathrm{PBS}$, which indicates that WP6 could form a stable complex with CPT/ HCPT during injection.

2.4. In Vitro Study. We then investigated the bioactivity of WP6-solubilized CPT and HCPT in vitro. It is crucial for the WP6 carrier to be biocompatible for any drug delivery application. The cytocompatibility of WP6 was checked in a normal cell line MC 3T3-E1 using a Cell Counting Kit-8 assay, and the results are presented in Figure S20. Very little cytotoxicity was observed for the WP6 systems with various concentrations. Even if the concentration of WP6 was as high as $500 \mu \mathrm{M}$, the cell viability was still above $90 \%$. Hence, WP6 has very good biocompatibility and shows great potential for biomedical applications.

To determine the antitumor activity of solubilized drugs in the WP6 systems, the cytotoxicity of WP6 systems containing CPT or HCPT against a tumor cell line Hela was also examined, and the results are shown in Figure 4. Because of the good biocompatibility of WP6 itself, the treatment of WP6 systems alone with Hela cells had no significant effect on the
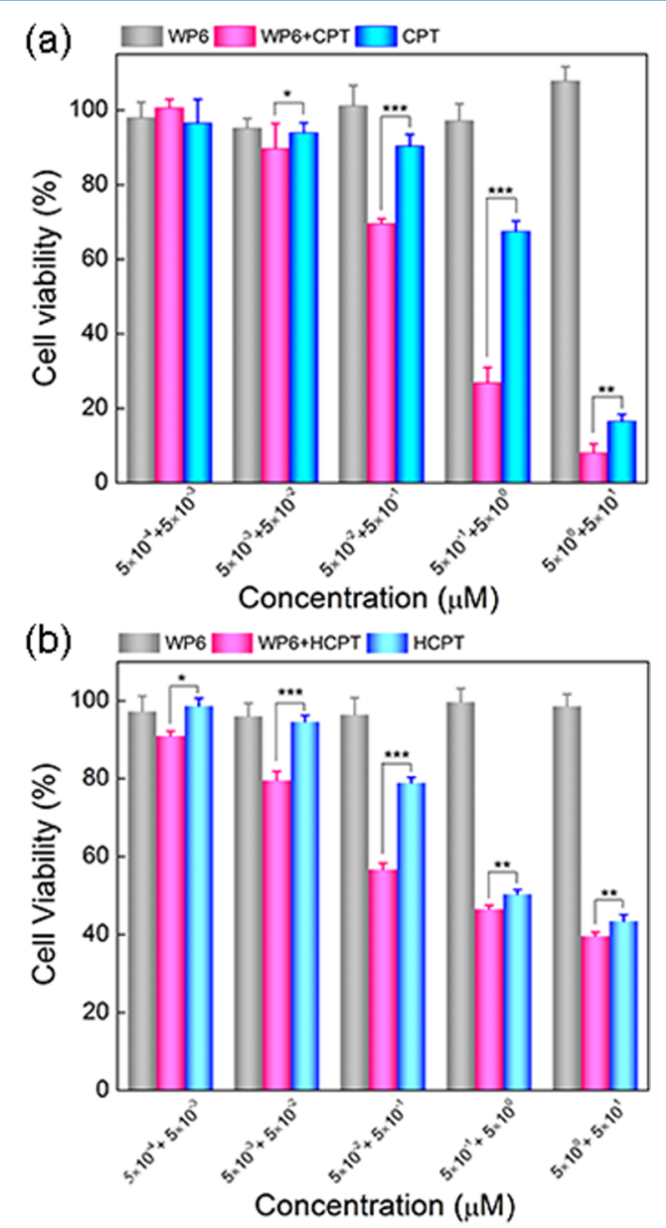

Figure 4. (a) In vitro cytotoxicity of WP6, CPT-loaded WP6, and free CPT against Hela cells as a function of CPT concentration. (b) In vitro cytotoxicity of WP6, HCPT-loaded WP6, and free HCPT against Hela cells as a function of HCPT concentration. The two values in the horizontal coordinate indicate the concentration of the drug (front) and WP6 (back), respectively. The blank control with culture medium only was set as $100 \%$ cell viability. Each point represents the mean \pm $\mathrm{SD} ; n=6$. Significant differences between the groups of free HCPT and HCPT-loaded WP6 determined by the Student's $t$ test are marked as the following criterion: $* p<0.05, * * p<0.01, * * * p<0.001$. cell viability. In sharp contrast, both the CPT-loaded WP6 system and the HCPT-loaded WP6 system exhibited an increased cytotoxicity with drug concentration. As shown in Figure 4, with a drug concentration of $5 \mu \mathrm{M}$, the viability of Hela cells reached 10 and $40 \%$ when treated with CPT-loaded WP6 and HCPT-loaded WP6, respectively. Calculated by the SPSS method, the half maximal inhibitory concentration $\left(\mathrm{IC}_{50}\right)$ of the CPT-loaded WP6 system was $0.1 \mu \mathrm{M}$, whereas that of the HCPT-loaded WP6 system was $0.5 \mu \mathrm{M}$. We also treated Hela cells with CPT or HCPT alone and calculated the $\mathrm{IC}_{50}$ to be 0.8 and $1.3 \mu \mathrm{M}$, respectively. It is obvious that the $\mathrm{IC}_{50}$ of WP6-solubilized CPT or HCPT is lower compared to that of the free drug. We also determined the influence of WP6 encapsulation toward bioactivity of the water-soluble antitumor drug TPT (Figure S21). The bioactivity of WP6-encapsulated TPT was slightly higher compared to that of the free drug at 5 $\mu \mathrm{M}$.

The cell assays indicate that WP6 is a biocompatible drug carrier to be used in vitro. By enhancing the solubility of CPT/ HCPT, WP6 is able to improve the cytotoxicity of the drugs. Because CPT/HCPT alone has very limited solubility in PBS, they are not soluble enough to be dosed via intravenous injection. With the solubility enhancement of WP6, CPT/ $\mathrm{HCPT}$ can reach a solubility of more than $1 \mathrm{mM}$ and may be used for chemotherapy in vivo.

\section{CONCLUSIONS}

In summary, water-soluble pillar[6] arene (WP6) dramatically enhances the solubility of CPT and HCPT. The study of hostguest chemistry confirms that WP6 could bind CPT family antitumor drugs with a stable complex formed between WP6 and CPT/HCPT. The bioactivity of CPT and HCPT was substantially improved with WP6 in vitro. The drug carrier WP6 was proven to have a low cytotoxicity and high biocompatibility. Our work demonstrates that WP6 and other water-soluble pillar[ $n]$ arenes have a great potential to be used for the formulation of poorly soluble drugs and active pharmaceutical ingredients (APIs).

\section{EXPERIMENTAL SECTION}

4.1. General Experimental. All reagents and solvents were purchased from commercial suppliers and were used without further purification. WP6 was prepared according to the procedure in the literature. ${ }^{25,35}$ Ultraviolet-visible (UV-vis) spectroscopy was performed on a Cary 100 Agilent UV-vis spectrometer. Fluorescence spectra were measured on a Shimadzu RF-1536 spectrometer. NMR spectra were recorded on an AVANCE III 400 spectrometer. Cell viability was measured on a Biotek ELx808 Microplate Reader.

4.2. Intrinsic Solubility of WP6. Excess WP6 was added into $\mathrm{NaD}_{2} \mathrm{PO}_{4}$ buffer $(10 \mathrm{mM}, \mathrm{pD}=7.4)$. The suspension was stirred at room temperature for $12 \mathrm{~h}$. The excess solid was removed with centrifugation. The concentration of WP6 in the supernatant was determined with ${ }^{1} \mathrm{H}$ NMR spectroscopy by comparing the integral of a known concentration of 1,3,5benzenetricarboxylic acid as the internal reference. The solubility of WP6 was calculated to be $33 \mathrm{mM}$.

4.3. Intrinsic Solubility of CPT/HCPT. Excess CPT was added into PBS. The suspension was stirred at room temperature for $6 \mathrm{~h}$. The excess solid was removed with centrifugation. The concentration of CPT was determined with UV-vis spectroscopy on the basis of a standard curve plotted 
with the UV-vis absorbance of CPT. The solubility of CPT was measured to be $5 \mu \mathrm{M}$.

Excess $\mathrm{HCPT}$ was added into $\mathrm{NaD}_{2} \mathrm{PO}_{4}$ buffer $(10 \mathrm{mM}$, pD $=7.4$ ). The suspension was stirred at room temperature for $3 \mathrm{~h}$. The excess solid was removed with centrifugation. The concentration of HCPT in the supernatant was determined with ${ }^{1} \mathrm{H}$ NMR spectroscopy by comparing the integral of a known concentration of 1,3,5-benzenetricarboxylic acid as internal reference. The solubility of HCPT was measured to be $29 \mu \mathrm{M}$.

4.4. Concentration of WP6-Solubilized Pharmaceutics. Excess CPT/HCPT was added into a solution of WP6 in $\mathrm{NaD}_{2} \mathrm{PO}_{4}(10 \mathrm{mM}, \mathrm{pD}=7.4)$ with a known concentration. The suspension was stirred at room temperature for $3 \mathrm{~h}$. The excess solid was removed with centrifugation. The concentration of CPT/HCPT in the supernatant was determined with ${ }^{1} \mathrm{H}$ NMR spectroscopy by comparing the integral with a known concentration of 1,3,5-benzenetricarboxylic acid as the internal reference. Each data point was performed in triplicate to calculate the deviation.

4.5. In Vitro Cytotoxicity of WP6 against Normal MC 3T3-E1 Cells. To determine the biocompatibility of WP6, the cytotoxicity of WP6 against normal MC 3T3-E1 was quantitatively detected by a Cell Counting Kit-8 (CCK-8) assay. MC 3T3-E1 cells were seeded into 96-well plates at $4 \times$ $10^{3}$ /well. Cells were first incubated in the atmosphere containing $0.5 \% \mathrm{CO}_{2}$ at $37{ }^{\circ} \mathrm{C}$ for $12 \mathrm{~h}$ before drug treatment. Next, the medium from the cells was aspirated and fresh medium $(200 \mu \mathrm{L} /$ well $)$ containing WP6 at varying concentrations $\left(5 \times 10^{-3}-5 \times 10^{2} \mu \mathrm{M}\right)$ was added as feed. After $24 \mathrm{~h}$ of incubation, the medium of each well was replaced with 200 $\mu \mathrm{L}$ of fresh medium containing $20 \mu \mathrm{L}$ of CCK-8 solution. Finally, the OD value of each well was detected at a wavelength of $450 \mathrm{~nm}$ by a Microplate Reader (Biotek ELx808) after $3 \mathrm{~h}$ of incubation. The relative cell viability was calculated to quantify the cytotoxicity, and the control group without treatment of any material except culture medium added was defined as $100 \%$ viability.

4.6. In Vitro Cytotoxicity of Combinations between WP6 and CPT/HCPT against Hela Cancer Cells. The cytotoxicity of solubilized CPT/HCPT in the WP6 systems against the Hela cancer cell line was examined by a CCK-8 assay. Hela cells were seeded in 96-plate well $\left(4 \times 10^{3} /\right.$ well $)$ and cultured for $12 \mathrm{~h}$. Then, the cells were treated with fresh medium $(200 \mu \mathrm{L} /$ well $)$ containing free $\mathrm{CPT} / \mathrm{HCPT}$, pure WP6, and CPT/HCPT-loaded WP6 systems at varying drug concentrations (for $\mathrm{CPT} / \mathrm{HCPT}$ and $\mathrm{CPT} / \mathrm{HCPT}$ loaded in WP6: $5 \times 10^{-4}-5 \times 10^{1} \mu \mathrm{M}$; for WP6 carrier: $5 \times 10^{-3}-5 \times$ $\left.10^{2} \mu \mathrm{M}\right)$ for another $24 \mathrm{~h}$. The detecting and analytical methods were the same as above-mentioned methods. The concentration of the test agent in 50\% cell viability was defined as $\mathrm{IC}_{50}$, and the SPSS software (IBM SPSS Statistics 21.0.0.0) was used to calculate the $\mathrm{IC}_{50}$ value.

4.7. Statistical Analysis. All of the cellular experiment results were expressed by their mean \pm SD (standard deviation). Comparisons between groups were analyzed by Student's $t$ test, and a $p$ value less than 0.05 was considered as the criterion of a statistically significant difference.

\section{ASSOCIATED CONTENT}

\section{S Supporting Information}

The Supporting Information is available free of charge on the ACS Publications website at DOI: 10.1021/acsomega.7b01032.
Methods for solubility measurement, ${ }^{1} \mathrm{H}$ NMR spectra, determination of the binding constant and supplementary in vitro data (PDF)

\section{AUTHOR INFORMATION}

\section{Corresponding Authors}

*E-mail: yu_lin@fudan.edu.cn (L.Y.).

*E-mail: dama@fudan.edu.cn (D.M.).

ORCID $\odot$

Lin Yu: 0000-0001-7660-3367

Da Ma: 0000-0002-5300-7500

Jiandong Ding: 0000-0001-7527-5760

\section{Author Contributions}

${ }^{\S}$ Y.L. and X.C. contributed equally.

\section{Notes}

The authors declare no competing financial interest.

\section{ACKNOWLEDGMENTS}

The authors are grateful to Fudan University (start-up grant), Natural Science Foundation of China (grant No. 51503042, 21672042, 51273217, and 21474019), and State Key Project of Research and Development (grant No. 2016YFC1100300) for financial support.

\section{REFERENCES}

(1) Wall, M. E.; Wani, M. C.; Cook, C. E.; Palmer, K. H.; McPhail, A. T.; Sim, G. A. J. Am. Chem. Soc. 1966, 88, 3888-3890.

(2) Giovanella, B. C.; Hinz, H. R.; Kozielski, A. J.; Stehlin, J. S.; Silber, R.; Potmesil, M. Cancer Res. 1991, 51, 3052-3055.

(3) Venditto, V. J.; Simanek, E. E. Mol. Pharmaceutics 2010, 7, 307349.

(4) Wani, M. C.; Nicholas, A. W.; Wall, M. E. J. Med. Chem. 1986, 29, $2358-2363$.

(5) Natsuka, K.; Nakamura, H.; Nishikawa, Y.; Negoro, T.; Uno, H.; Nishimura, H. J. Med. Chem. 1987, 30, 1779-1787.

(6) Burke, T. G.; Staubus, A. E.; Mishra, A. K.; Malak, H. J. Am. Chem. Soc. 1992, 114, 8318-8319.

(7) Wu, W.; Li, R.; Bian, X.; Zhu, Z.; Ding, D.; Li, X.; Jia, Z.; Jiang, X.; Hu, Y. ACS Nano 2009, 3, 2740-2750.

(8) Chang, G.; Ci, T.; Yu, L.; Ding, J. D. J. Controlled Release 2011, $156,21-27$.

(9) Yu, L.; Chang, G. T.; Zhang, H.; Ding, J. D. Int. J. Pharm. 2008, 348, 95-106.

(10) Ogoshi, T.; Kanai, S.; Fujinami, S.; Yamagishi, T.; Nakamoto, Y. J. Am. Chem. Soc. 2008, 130, 5022-5023.

(11) Cao, Y.; Hu, X.; Li, Y.; Zou, X.; Xiong, S.; Lin, C.; Shen, Y.; Wang, L. J. Am. Chem. Soc. 2014, 136, 10762-10769.

(12) Chang, Y.; Yang, K.; Wei, P.; Huang, S.; Pei, Y.; Zhao, W.; Pei, Z. Angew. Chem., Int. Ed. 2014, 53, 13126-13130.

(13) Hu, W.; Yang, H.; Hu, W.; Ma, M.; Zhao, X.; Mi, X.; Liu, Y. A.; Li, J.; Jiang, B.; Wen, K. Chem. Commun. 2014, 50, 10460-10463.

(14) Li, C.; Zhao, L.; Li, J.; Ding, X.; Chen, S.; Zhang, Q.; Yu, Y.; Jia, X. Chem. Commun. 2010, 46, 9016-9018.

(15) Li, S.; Zhang, H.; Xu, X.; Liu, Y. Nat. Commun. 2015, 6, No. 7590.

(16) Luo, L.; Nie, G.; Tian, D.; Deng, H.; Jiang, L.; Li, H. Angew. Chem. 2016, 128, 12905-12908.

(17) Ke, C.; Strutt, N. L.; Li, H.; Hou, X.; Hartlieb, K. J.; Mcgonigal, P. R.; Ma, Z.; Iehl, J.; Stern, C. L.; Cheng, C.; Zhu, Z.; Vermeulen, N. A.; Meade, T. J.; Botros, Y. Y.; Stoddart, J. F. J. Am. Chem. Soc. 2013, 135, 17019-17030.

(18) Sun, Y.; Guo, F.; Zuo, T.; Hua, J.; Diao, G. Nat. Commun. 2016, 7, No. 12042

(19) Ogoshi, T.; Ueshima, N.; Yamagishi, T.; Toyota, Y.; Matsumi, N. Chem. Commun. 2012, 48, 3536-3538. 
(20) Yakimova, L. S.; Shurpik, D. N.; Gilmanova, L. H.; Makhmutova, A. R.; Rakhimbekova, A.; Stoikov, I. I. Org. Biomol. Chem. 2016, 14, 4233-4238.

(21) Yu, G.; Zhao, R.; Wu, D.; Zhang, F.; Shao, L.; Zhou, J.; Yang, J.; Tang, G.; Chen, X.; Huang, F. Polym. Chem. 2016, 7, 6178-6188.

(22) Yu, G.; Yu, W.; Mao, Z.; Gao, C.; Huang, F. Small 2015, 11, 919-925.

(23) Joseph, R.; Naugolny, A.; Feldman, M.; Herzog, I. M.; Fridman, M.; Cohen, Y. J. Am. Chem. Soc. 2016, 138, 754-757.

(24) Han, C.; Ma, F.; Zhang, Z.; Xia, B.; Yu, Y.; Huang, F. Org. Lett. 2010, 12, 4360-4363.

(25) Yu, G.; Xue, M.; Zhang, Z.; Li, J.; Han, C.; Huang, F. J. Am. Chem. Soc. 2012, 134, 13248-13251.

(26) Wheate, N. J.; Dickson, K.-A.; Kim, R. R.; Nematollahi, A.; Macquart, R. B.; Kayser, V.; Yu, G.; Church, W. B.; Marsh, D. J. J. Pharm. Sci. 2016, 105, 3615-3625.

(27) Ma, D.; Hettiarachchi, G.; Nguyen, D.; Zhang, B.; Wittenberg, J. B.; Zavalij, P. Y.; Briken, V.; Isaacs, L. Nat. Chem. 2012, 4, 503-510.

(28) Yang, X.; Wang, Z.; Niu, Y.; Chen, X.; Lee, S. M. Y.; Wang, R. Med. Chem. Commun. 2016, 7, 1392-1397.

(29) Dong, N.; Xue, S.-F.; Zhu, Q.-J.; Tao, Z.; Zhao, Y.; Yang, L.-X. Supramol. Chem. 2008, 20, 663-671.

(30) Gavvala, K.; Sengupta, A.; Hazra, P. ChemPhysChem 2013, 14, $532-542$.

(31) Kuok, K. 1.; Li, S.; Wyman, I. W.; Wang, R. Ann. N. Y. Acad. Sci. 2017, 1398, 108-119.

(32) Okimoto, K.; Rajewski, R. A.; Uekama, K.; Jona, J. A.; Stella, V. J. Pharm. Res. 1996, 13, 256-264.

(33) Higuchi, T.; Connors, K. A. Adv. Anal. Chem. Instrum. 1965, 4, 117-210.

(34) Ma, D.; Zavalij, P. Y.; Isaacs, L. J. Org. Chem. 2010, 75, 47864795.

(35) Hu, X.; Chen, Z.; Chen, L.; Zhang, L.; Hou, J.-L.; Li, Z.-T. Chem. Commun. 2012, 48, 10999-11001. 